\title{
El concepto de cultura en la ensayística de Georg Simmel
}

\author{
[The Concept of Culture in Georg Simmel's Essays] \\ http://dx.doi.org/10.11606/1982-88372341153
}

\section{Francisco Manuel García Chicote ${ }^{1}$}

\begin{abstract}
This article analyzes Georg Simmel's concept of culture by reconstructing the genealogy of his theory within its historical, political, and economic context. It examines to what extent Simmel's ideas on value and division of labor cement his conception of cultural alienation. Finally, it argues that Simmel's cultural theory is significantly biased by a subjective theory of value, which entails apologetic traits.
\end{abstract}

Keywords: philosophy of life; alienation; subjective theory of value.

Resumen: El artículo indaga sobre el concepto de cultura en la ensayística de Georg Simmel. Para ello, examina ante todo el significado de la noción al interior del campo intelectual alemán en cuyo seno se desarrollaron las formulaciones teóricas del filósofo berlinés. A continuación, se abordan los factores fundamentales del concepto de cultura en Simmel: sus aspectos práctico y crítico. Se analiza en qué medida estos factores se hallan condicionados por una teoría subjetivista del valor y por un concepto ahistórico de división del trabajo. Se concluye que la teoría cultural de Simmel se encuentra condicionada significativamente por un individualismo metodológico, lo que conlleva elementos apologéticos.

Palabras clave: filosofía de la vida; alienación; teoría subjetivista del valor.

\section{Introducción}

Se persigue en estas líneas el siguiente objetivo: echar luz sobre un concepto que cimenta la crítica de la modernidad de quien es considerado, por lo menos en los últimos treinta años, uno de los fundadores de la sociología moderna. Se trata, pues, de Georg Simmel y de su concepto de cultura. Dos motivaciones inmediatas impulsan esta empresa. De una parte, aparece a primera vista el potencial crítico de la teoría cultural del filósofo berlinés: reclama para sí tanto una perspectiva plebeya -pues la lógica cultural anima cualquier

\footnotetext{
${ }^{1}$ Universidad de Buenos Aires, Instituto de Filología y Literaturas Hispánicas "Dr. Amado Alonso", Sección de Literaturas en Lenguas Extranjeras, 25 de mayo, 217, primer piso, Ciudad Autónoma de Buenos Aires, C1002ABE, Argentina. E-mail: fgchicote@ gmail.com. ORCID: 0000-0002-3267-390X
}

\section{(cc) BY-NC}

Pandaemonium, São Paulo, v. 23, n. 41, set.-dez. 2020, p. 153-175 
García ChICOTE, F. M. - El concepto de cultura en la ensayística de Georg Simmel

manifestación del espíritu sin importar la adscripción de clase, de casta, o de grupo de poder- como un punto de partida cuestionador, profundamente sensible al problema por antonomasia de la radicalidad del ser humano, esto es, su alienación, el hecho de que su falta de libertad es autoproducida. Sobre este carácter crítico de la teoría cultural de Simmel podría remitirse alguna línea de quien le adjudicara hacia mediados de la década de 1980 el epíteto de "primer sociólogo de la modernidad”. Señala David Frisby (2013: 24) en los albores del "Simmel-Renaissance" que la obra clave de Simmel -su Filosofía del dinero- presenta "una teoría de alienación cultural que, a primera vista, parece muy cercana a la del joven Marx"; así, en la última parte del libro de 1900 “-que destaca por derecho propio como uno de los primeros análisis sociológicos de los modos de vivir y experimentar la modernidad-Simmel establece las bases para una teoría de alienación en el contexto del desarrollo cultural moderno" (FRISBY 2013: 24). El alcance de esta teoría constituye una signatura idiosincrásica de su pensamiento; acaso, tal como recuerda Jürgen Habermas (1996: 405), uno de los motivos de su dificultosa inserción en la academia alemana: ${ }^{2}$

Lo que lo distanciaba a Simmel del mundo académico era su mentalidad, caracterizada por una percatación sensible de las atracciones típicas de su época, de las innovaciones estéticas, de los movimientos de disposición espirituales y los cambios de orientación en las actitudes metropolitanas con relación a la vida, y de las transformaciones en las inclinaciones subpolíticas y los fenómenos cotidianos, apenas tangibles, difusos y movedizos. ${ }^{3}$

La teoría simmeliana, supuestamente "atractiva para aquellos que buscaban un camino hacia un marxismo que no se hallara lastrado por el rígido marco de basesuperestructura" (FRISBY 2013: 28), abreva en una concepción descentrada, subjetivista, fortuita y evanescente de la existencia social cuyas semejanzas con el giro posmoderno no demoraron en ser señaladas. El otro impulso para una revisión de la teoría cultural de Simmel estriba precisamente no ya en su adecuación con su objeto, sino en la sugerente afinidad que mantiene, a pesar de su ya avanzada edad, con formulaciones propias del capitalismo tardío. Remitiéndose a la centralidad que presentaría en la obra de Simmel la fragmentación de la experiencia, el propio Frisby (1992: 14) afirma en otro contexto que el filósofo "prefiguró en algunos aspectos su acentuación en la llamada posmodernidad". ${ }^{4}$

\footnotetext{
${ }^{2}$ Recién en 1914, a los 56 años de edad y luego de una serie de intentos fallidos, obtuvo Simmel una cátedra. ${ }^{3}$ Cuando la versión referida la sección de bibliografía no se encuentra en español, la traducción es nuestra. ${ }^{4}$ La misma afinidad es advertida, ya tres décadas más tarde, por Karl-Siegbert Rehberg (2018: 304) al definir la idiosincrásica dialéctica de sujeto y objeto que funda el concepto simmeliano de cultura: "En tiempos de una abierta y al mismo tiempo subjetivización, el relacionismo de Simmel y su conexión entre
} 
García ChICOTE, F. M. - El concepto de cultura en la ensayística de Georg Simmel

Las siguientes páginas dividen su propósito en dos partes. Un análisis del concepto simmeliano de cultura debería ante todo recuperar los elementos socio-históricos básicos del significado del término: aquellos sobre los que la teoría de Simmel se apoya insoslayablemente. Esto resulta tanto más importante cuanto que la pregunta por la actualidad debería comenzar por la constatación de que el término estaba lejos de significar lo que hoy en día se entiende por él. ${ }^{5}$ Se intentará dar con una definición esquemática de tres rasgos superpuestos, cada uno más concreto que el anterior: la cultura 1. como ámbito de los valores; 2. como forma de la autoconciencia de la burguesía en ascenso; 3. como forma específica del desarrollo alemán al capitalismo: la cultura como vía prusiana. Luego, en segunda instancia, se analizará el concepto de cultura de Simmel a la luz de su teoría del valor para comprender las implicancias que dicha teoría, anclada en una visión insoslayablemente subjetivista, imprime sobre el concepto de cultura. Se sostiene que la teoría cultural de Simmel descansa sobre una concepción subjetivista y ahistórica de la relación sujeto-objeto: su alfa y omega es el individuo particular burgués que se determina a sí mismo. Así, se cercenan las posibilidades críticas del concepto y asoman en cambio trazos apologéticos.

\section{Cultura en el campo intelectual alemán}

En 1952, Alfred Kroeber y Clyde Kluckhohn (1952: 41) registraron 154 diferentes definiciones modernas del concepto, que categorizaron "con gran artificialidad" en seis grupos semánticos, más un conjunto de siete definiciones "incompletas". Mucho más simple es el esquema dual ofrecido en 2002 por Dirk Baecker: "cultura" implica una noción o bien "estética" o bien "empírica". Esta última pretende ser ante todo axiológicamente neutral: se concentra en un grupo humano al describir su origen, su estructura y sus funciones con arreglo a sus modos peculiares de interacción entre

\footnotetext{
relaciones recíprocas personales y patrones de orden suprapersonales permiten hoy entrever una perspectiva - enfriada hoy empero desde el punto de vista de la filosofía de la historia- como la que fue llevada a cabo en los descentramientos posmodernos".

${ }^{5}$ Paul Nolte (1998: 235), en su fundamentación sobre la actualidad de la teoría sociológica de Simmel, afirma que el concepto de cultura de este "va mucho más allá de un concepto idealista de cultura y sigue más bien una comprensión moderna, amplia que no ha tenido problemas en incluir la cultura material y dentro de ella incluso los objetos aparentemente banales de la vida cotidiana". Al sopesar, en 1983, los alcances de la actualidad de su pensamiento llega Habermas a una conclusión difícilmente avenible con la de Nolte: "Es la noción neokantiana de cultura lo que señala aquello que nos separa de Simmel" afirma Habermas (1996: 407).
}

Pandaemonium, São Paulo, v. 23, n. 41, set.-dez. 2020, p. 153-175 
García ChICOTE, F. M. - El concepto de cultura en la ensayística de Georg Simmel

individuos. El concepto estético de cultura, en cambio, "impulsa esta comprensión empírica con intenciones humanas. En un nivel estético, el concepto de cultura aspira al devenir completamente cultural de la cultura" (BAECKER 2002: 510). De acuerdo con Baecker, los abordajes estéticos pueden adoptar o bien una agenda universal e interesarse entonces en la humanización de la humanidad (las Cartas de Schiller o el Wilhelm Meister de Goethe serían casos en cuestión), o bien una agenda particular, "ecológica", en la que "cultura" es entendida como una "identidad cohesiva de organismo, pysche y comunicación, que solo puede concebirse 'ecológicamente' y percibirse solo ‘estéticamente"”. Heinrich Rickert (1943: 54), un filósofo neokantiano que desempeñó un papel considerable en la investigación que condujo a Filosofía del dinero, definió el elemento constituyente de la cultura estética: el valor. "Sin un punto de vista afianzado en los valores”, dice en 1899 al proponer cambiar la designación de ciencias del espíritu por ciencias de la cultura, "sin un punto de vista que separa los bienes de otras realidades sin valor, es imposible hallar una distinción aguda entre naturaleza y cultura”. No es de extrañar que la perspectiva que abre una teoría cultural estética ponga en el centro de sus intereses el problema de la alienación. O, en otras palabras: las teorizaciones axiológicas de la cultura presentan como tarea principal el examen de aquello que obstaculiza la humanización. La teoría cultural estética es por necesidad intencionalmente crítica, tan pronto como deja de aparecer como crítica, cesa de ser teoría cultural. Análogamente, nociones tales como "alienación", "humanización”, corrompen las teorías empíricas de la cultura tan pronto como cesan de referirse a fenómenos simbólicos estrictamente observacionales y pretenden actuar metódicamente en tanto categorías fundamentales.

Esta variante axiológica del concepto de cultura tiene un origen histórico y político preciso: comienza con la consolidación de la sociedad burguesa. No es de extrañar que el concepto asuma pues la forma de la conciencia típica de la clase que lo impulsa en tanto reflejo de sí. ${ }^{6}$ En 1911, György Lukács, que había asistido al seminario de Simmel durante 1907 y 1908, señaló la fragilidad conceptual detrás de la fachada de la cultura. Así aparece en su ensayo de 1911 "Cultura estética” (LUKÁCS 2015: 185-200), que marca un buen contrapunto con el trabajo de Simmel, publicado el mismo año, "Sobre el concepto y la

\footnotetext{
${ }^{6}$ El ya citado Baecker (2002: 512) y Georg Bollenbeck (1996: 55-61) adjudican un impulso decisivo para la carga conceptual moderna del término a la segunda edición de la obra de Samuel von Pudendorf De jure naturae et gentium, de 1684. Para ambos críticos, decisivo es el hecho de que Pudendorf vea la cultura ya no como un proceso de "cultivación" moral, sino como una esfera propia, cualitativamente distinta de la naturaleza, en la que se incluyen de manera central elementos políticos.
} 
García ChICOTE, F. M. - El concepto de cultura en la ensayística de Georg Simmel

tragedia de la cultura". El joven húngaro parte de la contraposición, ciertamente extendida por entonces, entre la cultura en tanto dominio de realización interior, auténtica y espiritual y, por el otro, la civilización como ámbito de lo material, lo exterior, lo meramente técnico. Para Lukács (2015: 188-194), "la mentira fundamental de la cultura estética" consistía precisamente en aquello que compartía con otros conceptos clave para la formación de la autoconciencia burguesa: el carácter adialéctico de su idealismo. La teoría cultural asume que la vida auténtica puede ser alcanzada únicamente en el ámbito de la interioridad (por medio de la cultivación del alma, etc.), sin poder así admitir la dependencia de este ámbito respecto de lo exterior, lo que significa no poder reconocer el condicionamiento histórico y social del alma. 25 años más tarde, y retomando la crítica lukácsiana de las antinomias del pensamiento burgués de 1923, Herbert Marcuse (1970) definió la cultura como un dominio inmaterial de existencia en el que las promesas sociales alguna vez hechas por el liberalismo burgués hallarían su concretización exclusiva. Marcuse (1970) llama, de hecho, esta esfera de humanización puramente espiritual "cultura afirmativa" y sostiene que los valores universales que propone derivan de la prevalencia del trabajo abstracto en el capitalismo. En la medida en que la teoría cultural propone vías de humanización, constituye una feroz crítica de los procesos alienantes que penetran la cotidianidad capitalista. En la medida, sin embargo, en que necesariamente desconoce su consanguinidad con lo material, con la civilización, la teoría cultural no solo tiene una tendencia a comprender la cultura como algo de hecho universal y eterno, sino que también deja intacta la miseria de la vida. Detrás de sus elementos críticos, la cultura afirmativa esconde pues una verdadera función social: la reproducción de la miseria actual. Una vez que se tornó evidente que la burguesía no podría honrar el rol universalmente redentor que se había imputado en la historia mundial, se elevaron "demandas acusadoras", a las que

la burguesía dio una respuesta decisiva: la cultura afirmativa. Esta es, en sus rasgos fundadores, idealista. A la penuria del individuo asilado responde con la humanidad universal, a la miseria corporal, con la belleza del alma, a la servidumbre externa, con la libertad interna, al egoísmo brutal, con el reino de la virtud del deber. Si en la época de la lucha ascendente de la nueva sociedad, todas estas ideas habían tenido un carácter progresista destinado a superar la organización actual de la existencia, al estabilizarse el dominio de la burguesía, se colocan, con creciente intensidad, al servicio de la represión de las masas insatisfechas y de la mera justificación de la propia superioridad: encubren la atrofia corporal y psíquica del individuo. (MARCUSE 1970: 52)

Hacia 1940, esta función ideológica fue denunciada por Bertolt Brecht (1995) en la parábola "Si los tiburones fueran hombres". Allí se describe un mundo imaginario en 
García ChICOTE, F. M. - El concepto de cultura en la ensayística de Georg Simmel

el que los tiburones asumen comportamientos humanos; los pececillos, pues, ya no son instintivamente comidos por los selaquimorfos, sino que devienen presa de un refinado y minucioso proceso ideológico de sometimiento, en el que ellos mismos participan activamente de su propia ruina. La parábola termina así: "En suma, habría ante todo una cultura en el mar, si los tiburones fueran hombres" (BRECHT 1995: 448). ${ }^{7}$

No es casual que estas críticas emanen de contextos alemanes. En los comienzos de su desarrollo, hacia la segunda mitad del siglo XVIII, el concepto de cultura configuraba una constelación de nociones (entre otros: formación, personalidad, ilustración) que resultaron decisivas para la constitución de una autoconciencia intelectual de sectores medios burgueses, en su lucha contra la nobleza cortesana. El carácter ilustrado del concepto, vinculado a la perfectibilidad del ser humano, aparece ya en un ensayo de 1784 de Moses Mendelssohn (1974: 4), en el que se afirma que "formación, cultura e ilustración son modificaciones de la vida social, el resultado de la industria y los esfuerzos de la humanidad para mejorar su condición social". Sobresale aquí una signatura particular del modo en que los intelectuales alemanes de la segunda ilustración “rechazaron” (BOLLENBECK 1996: 117) el pesimismo cultural de Rousseau. Martín Koval (2018: 37) señala "una tergiversación" o al menos "una interpretación particular" del concepto rousseauniano de perfectibilidad, tal como aparece en su Discurso sobre el origen y los fundamentos de la desigualdad entre los hombres, de 1754. Mientras que, para Rousseau, la facultad de perfeccionarse, la capacidad de crear una cultura, aparta al hombre de su estado natural y confiere el fundamento de la infelicidad y los vicios, la recepción alemana durante el último tercio optó por concebir la perfectibilidad como algo “exclusivamente positivo" (Koval 2018: 40). Se procuró, en efecto, encontrar una vía estética para la formación del individuo, lo que, tal como afirma Hans Robert Jauss (1995: 78) “permitió dar un carácter positivo al diagnóstico de Rousseau de la autoalienación del hombre y la sociedad".

Aquí vale destacar, junto al carácter positivo de la noción, su rasgo estrictamente apolítico. Demasiado débil y dispersa para conformar una esfera pública, explícitamente

\footnotetext{
${ }^{7}$ Una década más tarde, en 1951, Theodor W. Adorno (2001: 41-42) intenta discernir, en el concepto de cultura, un factor ideológico de un factor verdadero y críticamente auténtico. En la Miniatur 22 de Mínima Moralia, "cultura" es ciertamente asociada con una "mentira" que hace "creer en una sociedad humanamente digna que no existe; que oculta las condiciones materiales sobre las que se levanta todo lo humano; y que, con apaciguamientos y consuelos, sirve para mantener con vida la perniciosa determinación económica de la existencia”. Sin embargo, dado que aquello que ella niega, el cambio libre y legal, es precisamente "la mentira", favorece a la verdad.
}

Pandaemonium, São Paulo, v. 23, n. 41, set.-dez. 2020, p. 153-175 
García ChICOTE, F. M. - El concepto de cultura en la ensayística de Georg Simmel

proscripta de la participación en las cortes, desprovista de cualquier elemento masivo al que interpelar, la intelectualidad ilustrada alemana formó una constelación axiológica en torno a la interioridad, a lo "puramente espiritual", en la que la cultura desempeñaba un rol central. Se trata, en palabras de Norbert Elias (2009: 97), exclusivamente de "ideas, sentimientos", desvinculados de cualquier implicancia políticamente concreta:

La constitución de los pequeños estados absolutistas de aquella sociedad no ofrecía oportunidad alguna para tal actividad política. Los elementos burgueses adquirían cada vez una mayor conciencia; sin embargo, la red de los Estados absolutos no se conmovía. Los elementos burgueses estaban excluidos de la participación política; todo lo más que podían hacer de modo autónomo era "pensar y escribir poesía", pero no actuar. En esta situación, la actividad literaria venía a ser una especie de substitutivo de la acción política. En ella se expresan de modo más o menos oculto los nuevos sentimientos, así como el descontento con la situación existente. En este ámbito que, hasta cierto punto, dejaba en libertad al Estado absoluto, la nueva generación de clase media, provista de sus nuevos sueños y de sus ideales opositores, se enfrentó a los ideales cortesanos, y lo hizo en idioma alemán.

La marcada apoliticidad, resultante de una necesidad objetiva propiamente alemana y esencialmente diversa a los desarrollos inglés y francés, se convierte en virtud cuando, un siglo más tarde, la burguesía consolida su dominio político y económico en avenencia con el absolutismo feudal de los Junkers. Poco queda ya de la primigenia estructura -ciertamente problemática- ilustrada: "cultura" asumió en Alemania hacia el fin del siglo XIX elementos apologéticos del modo peculiar y tardío en que el país se integró a las formas políticas y económicas de la sociedad burguesa (cf. ELIAS 2009: 85115; PETER 2016: 100). Lukács (1972: 327-328) y Kroeber y Kluckhohn (1952: 28) señalan que la consolidación de la noción de cultura al interior del campo intelectual alemán se halla profundamente relacionada con la desintegración del hegelianismo, al punto que una noción no dialéctica de cultura se impuso sobre la de "espíritu", con fuertes reminiscencias, en última instancia, de ideas racionalistas y humanistas de praxis y totalidad. A partir de la integración reaccionaria, "desde arriba", de la nación alemana, "cultura" constituye una noción decisiva en la así llamada "Filosofía de la vida" y tiene tres aspectos definitorios. En primer lugar, "cultura" es, en oposición a "civilización", el tesoro distintivo de una supuesta alma alemana, involucra tanto las expresiones como la expresividad de una nación forjada de un modo único por elementos tanto históricos como metafísicos. En contraposición, “civilización” posee más bien un aspecto “acumulativo", procedimental, empírico y superficial (cf. MERTON 1936: 110): se la asocia con Inglaterra y Francia y sus atributos políticos, económicos e intelectuales 
GarCíA CHICOTE, F. M. - El concepto de cultura en la ensayística de Georg Simmel

(empirismo inglés, racionalismo francés, republicanismo, individualismo, liberalismo). ${ }^{8}$

En segunda instancia, dado su carácter único y su vínculo con un modo de vida supuestamente inmediato, anterior a la conceptualización formal, la cultura no solo exige para sí una coherencia irracional, sino que también desarrolla una crítica irracionalista de lo socialmente concreto. Al denunciar la cosificación en los procesos económico e intelectual, la teoría cultural ofrece en efecto una crítica del "frío entendimiento" del racionalismo, pero confunde instrumentalidad formal y categorial con razón, y legitima así modos de comprensión "inmediatas", "intuitivas”, cuya ocurrencia es, desde el punto de vista de su conceptualización, contingente. El elitismo irracionalista que resulta de esta crítica se vincula con las nociones de secreto, misterio y latencia, tan expandidas en el círculo de Stefan George. El tercer aspecto del desarrollo alemán del concepto de cultura es su superioridad. Al ser la cultura especialmente alemana $y$ la manera exclusivamente efectiva de eliminar las amenazas alienantes de la civilización, se sigue que Alemania goza, gracias a la cultura, de una posición de superioridad frente a otras naciones (primariamente Francia e Inglaterra, luego “América” y las repúblicas soviéticas del este). Esto ya aparece en el ensayo de Dilthey (1950: 162) sobre Goethe; este, presumiblemente la fruta más acabada del suelo germánico, encarnaría una síntesis descosificadora del "solipsismo" de Rousseau y el "empirismo" de Shakespeare. En última instancia, la idiosincrasia alemana se nutre del carácter cultural de su esencia: mientras que la civilización puede ser comprendida como la expresión de un espíritu fosilizado, cuya expresividad es meramente aditiva, superflua, cuantitativa, la significancia de la cultura apunta a un espíritu vivo que aún no ha desempeñado su parte decisiva en la historia universal.

A la luz del desarrollo particular del concepto en Alemania, y siguiendo la línea crítica de Lukács en 1911, Adorno examinó la función cultural del genocidio nazi. En 1966, afirma en efecto que si la cultura "aborrece el hedor es porque ella misma hiede; porque, como dice Brecht en un magnífico pasaje, su palacio está hecho de mierda. [...]

\footnotetext{
${ }^{8}$ El famoso ensayo de Wilhelm Dilthey acerca de Goethe, publicado por primera vez en 1877 y luego integrado a Vida y poesía (DILTHEY 1950), muestra los términos concretos de esta combinación de esencia e historia. Dilthey divisa un impulso inherentemente alemán hacia la armonía en una época en la que los poderes corruptores y disolventes de la Revolución Francesa amenazaban con exterminar todos los organismos vivientes. Define además el tipo concreto de sujeto histórico que mejor se corresponde con los valores culturales: la personalidad. Una personalidad es cualquier individuo que, en virtud de una misteriosa relación con el mundo (esto es, una "vivencia", que implica la eliminación del entendimiento categorial y la emergencia de una relación orgánica, irracional, embriagante entre sujeto y objeto) ha ganado una interioridad armoniosa y puede llevar a cabo en la historia (en arte, en la política) el impulso latente del alma alemana.
}

Pandaemonium, São Paulo, v. 23, n. 41, set.-dez. 2020, p. 153-175 
GarCíA CHICOTE, F. M. - El concepto de cultura en la ensayística de Georg Simmel

En esos santuarios del espíritu, en la pretensión enfática de su autarquía es precisamente donde radica la mentira" (ADORNO 2002: 333).

\section{Aspectos del concepto en Simmel}

Cuando Simmel habla de cultura, no hay una referencia a una versión neutral, empírica del concepto, sino a un complejo de nociones axiológicas que posee cuatro características básicas: 1. remite a la humanización del hombre, 2. asume por tanto una posición crítica respecto de la situación actual, 3. es suspicaz respecto de la facultad redentora de la razón, 4. despliega por momentos una idea política peculiar de Alemania. De ningún modo empero han de constituir las premisas señaladas arriba una definición de lo que Simmel entiende por cultura; antes bien, deben considerarse como una abstracción esquemática de sus factores condicionantes. Los trazos fundamentales del concepto de cultura en Simmel son dos: sus elementos práctico y crítico; ambos reciben una coloración especial del concepto simmeliano de valor.

Las reflexiones de Simmel sobre la cultura ocupan centralmente su ensayística de las primeras décadas del siglo pasado. Si bien se perciben desarrollos un tanto originales en los últimos años - por ejemplo, que analizan la implicancia de la Primera Guerra Mundial o integran un vitalismo marcadamente metafísico al problema $-{ }^{9}$ que testimonian una tendencia general del pensamiento del filósofo hacia un subjetivismo radical, los parámetros teóricos básicos se establecen ya en Filosofía del dinero, de 1900. Decisivo es el tratamiento que allí recibe el concepto de valor. En concordancia con el giro subjetivista que caracteriza el proceso de disolución de la escuela ricardiana y, naturalmente, con los desarrollos neokantianos de la academia alemana, para Simmel el valor no resulta de una propiedad del objeto, sino que proviene de una facultad espiritual irreductible a este. ${ }^{10}$ Se trata de un "fenómeno primigenio", subjetivo en la medida en que

\footnotetext{
${ }^{9}$ Para una profundización de estos elementos, véanse los trabajos de Peter (2016) y Becher (2008).

${ }^{10}$ Para Frisby, existe una estrecha cercanía entre la teoría simmeliana del valor y los aportes de Carl Menger y Eunge Böhm-Bawerk (FRISBY 2013: 14; 1985: 59-60). Cabe aclarar en este punto que el propio Simmel se distancia de la comprensión marginalista según la cual el valor se halla condicionado por la utilidad que suscita al sujeto consumidor la última unidad disponible de un bien (cf. SIMMEL 2013: 82-87). Esta diferencia respecto de "los inútiles cálculos de los utilitaristas por un lado y de la escuela austríaca por el otro" fue señalada oportunamente por George H. Mead en 1900 (2003, p. 302) y también, aunque un tanto más morigerado, por el propio Gustav Schmoller (2003: 283) en la reseña que le dedicara a la obra de Simmel en 1901. En contextos más contemporáneos, Alex T. Paul (2018: 599-600) habla de "diferencias esenciales" respecto de las posiciones neoclásicas y Natàlia Cantó Milà (2005) subraya los aspectos originales de la contribución a la teoría del valor. Como Simmel no incurriría en la hipóstasis del individuo
} 
GarCíA CHICOTE, F. M. - El concepto de cultura en la ensayística de Georg Simmel

“en ningún caso es el valor de uno de los 'atributos' de las cosas, sino un juicio sobre ellas que el sujeto formula": cuando el "alma" abandona una actitud desinteresada respecto de los contenidos de la realidad, los capta en un "orden completamente autónomo" (SIMMEL 2013: 44, 46 y 42). La esfera axiológica es propiedad exclusiva del ser espiritual ${ }^{11}$ y surge precisamente a partir de la separación entre el sujeto y objeto; ${ }^{12}$ de ahí que, tal como señala Michael Heinrich (2017: 66), la forma del valor simmeliana "no solo es independiente del intercambio, sino también en realidad de toda sociedad". De ahí también que Simmel (2013: 69-73) comparta con el giro subjetivista de las últimas décadas del siglo XIX el carácter en última instancia ahistórico de la forma propuesta de valor: el modelo heurístico primitivo que se esboza para echar luz sobre la fijación del valor parte de la "economía natural", del trueque entre dos individuos privados o incluso de un imaginario "productor aislado", en quien la relación con la naturaleza se consuma en la forma de un intercambio de bienes efectuado según la ecuación de "deseo" y "sacrificio". La posición del individuo no como punto de llegada sino como alfa metodológico, la preeminencia de la categoría de intercambio por encima de la de producción y la afirmación de una facultad valorativa no objetiva son factores que, a pesar de la idiosincrásica coloración que asumen en la obra de Simmel, indican vínculos no menores del pensamiento de este con el derrotero del pensamiento filosófico en la disolución del hegelianismo y, además, con el desarrollo de la teoría económica burguesa de finales del siglo XIX. ${ }^{13} \mathrm{Al}$ respecto de esta última, encarnada en la "escuela austríaca",

particular empírico, su distancia respecto de los aportes de la escuela austríaca sería significativa (CANTó MILÀ 2005: 172). Sin menospreciar estas divergencias, lo que interesa aquí fundamentalmente es el carácter en última instancia subjetivista de la posición de valor, que, por un lado, constituye un comportamiento intelectual de época y, por el otro, tendrá implicancias significativas en la concepción simmeliana de la cultura.

${ }^{11}$ Se ve aquí la impronta neokantiana y, en particular, la de Heinrich Rickert en el concepto simmeliano de valor. En Ciencia cultural y ciencia natural, de 1899, Rickert (1943: 54) afirma que "[1]os procesos culturales habrán de considerarse realmente, no solo con referencia a un valor, sino al mismo tiempo con referencia a un ser psíquico de los valores; porque los valores son valorados por seres psíquicos". Simmel ya había leído el libro a finales de 1898, pues el 31 de diciembre le escribe a Rickert que le "alegra poder concordar en casi todo. Solo que me parece que esta constatación metodológica requiere aún una fundamentación adicional en lo epistemológico, incluso en lo metafísico. Sobre algunas de las cuestiones tratadas por usted me he explayado en los capítulos ya concluidos de mi Filosofía del dinero" (SIMMEL 2005a: 319-320).

${ }^{12}$ El ya citado Paul (2018: 599) afirma: "Valor llama Simmel justamente a aquella distancia que se establece entre el sujeto que deviene tal y el objeto representado".

${ }^{13}$ En un reciente trabajo, Spiros Gangas (2019: 9) argumenta que hay suficientes afinidades entre la obra tardía de Simmel -ante todo su parcialmente póstuma Intuición de la vida-y las interpretaciones de Herbert Marcuse sobre Hegel y la filosofía de Dilthey como para buscar, por un lado, elementos críticos de la filosofía simmeliana que no se avengan con el carácter reaccionario de la Kulturkritik y, por el otro, continuidades significativas entre Simmel, Hegel y Marx, al punto que sería posible "interpretar la categoría de vida [simmeliana] dentro del marco racionalista forjado por Marx y Hegel". Independientemente del

Pandaemonium, São Paulo, v. 23, n. 41, set.-dez. 2020, p. 153-175 
GarCíA CHICOTE, F. M. - El concepto de cultura en la ensayística de Georg Simmel

Isaac Illich Rubin (2017) denunció en un artículo de 1926 para la Gran enciclopedia soviética que su punto de partida no eran las relaciones socio-objetivas entre las personas, sino la psicología y los motivos de individuos particulares. Se estudia así "la 'actividad económica' en general, independientemente de la forma histórica de la economía”, como el resultado de un "agregado de Robinson Crusoes", cuyas "experiencias psicológicas y motivaciones en tanto consumidores particulares" son las "fuerzas motrices de la sociedad" (RUBIN 2017: 440s.). Una ponderación sugerentemente afín suscita el caso de Filosofía del dinero en el análisis de Heinrich Brinkmann (1974). Brinkmann (1974: 117) caracteriza al sujeto económico primigenio de Simmel como "Robinson trascendental", un individuo dotado de todas las características propias de la existencia en el capitalismo desarrollado: "Lo que Simmel emprende aquí en intenciones metodológicas acaba en una ontologización de determinados modos de comportamientos humanos que fueron desarrollados bajo determinadas condiciones sociales".

No se trata aquí de menospreciar la viva y heterogénea complejidad de la obra de Simmel, ni de encasillarlo como mero exponente de una "economía política vulgar" que se detiene adialécticamente en el nivel de la mera apariencia. En cambio, se intenta comprender en qué medida esta teoría del valor condiciona el concepto de cultura. Y lo hace al menos desde dos perspectivas. Por un lado, la insuperable brecha entre el mundo axiológico/espiritual y el mundo objetivo/natural influye en lo que Simmel entiende como "concepto universal de cultura": un modo específico de actividad que tiene por objeto el desarrollo del individuo "más allá del estado de naturaleza" hacia la formación armoniosa de una personalidad (SIMMEL 2013: 532-533). En esta actividad se impone siempre, de manera inevitable, el conflicto entre el carácter objetivo de los medios y la esencia subjetivamente autónoma de los valores. Por otro lado, el individualismo

\footnotetext{
valor metodológico del proceso que conduce a tal conclusión -es sugerente que el análisis casi no contraponga a Simmel con Hegel y Marx directamente, sino que lo haga en la mayoría de las veces a través de las interpretaciones que Marcuse hizo de estos dos; asimismo resulta llamativo que el trabajo no historice las categorías que le sirven de ejes de comparación antes de usarlas como tales-, es cierto que una lectura hegelianizante de Simmel fue propuesta hace más de cuatro décadas por Petra Christian, (1978) cuya "olvidada" (GANGAS 2019: 6) monografía ve en el concepto simmeliano de reciprocidad similitudes con el pensamiento dialéctico de Hegel. No se desconocen en el presente trabajo tendencias generales del pensamiento alemán moderno que actúan como motores tanto en Marx como en Hegel, Simmel y muchos otros: la crítica de la alienación moderna, la crítica del individuo particularista, la pulsión hacia la totalidad, asuma esta caracteres orgánicos o remita a relaciones de condicionamientos recíprocos, etc. (ciertamente, la persistencia de estos elementos no implica que las conceptualizaciones se mantengan estables; tal es el caso, como se ha señalado arriba, del concepto de cultura; para el problema, cf. por ejemplo GAY 1992: 75101). Se trata en cambio de echar luz sobre ciertas particularidades de la noción de cultura de Simmel que se relacionan con su concepto de valor, que arraiga a su vez en formas intelectuales propias del desarrollo social de la segunda mitad del siglo XIX.
}

Pandaemonium, São Paulo, v. 23, n. 41, set.-dez. 2020, p. 153-175 
García ChICOTE, F. M. - El concepto de cultura en la ensayística de Georg Simmel

metodológico que cimenta la propuesta de Simmel permite una determinación "universal" del concepto en el que el mundo social se despliega a partir de un particularismo no mediado: al concebir la emergencia histórica de la división técnica del trabajo al interior de la empresa capitalista como la forma normal de la división social del trabajo, Simmel le imputa a esta la ruina del individuo multilateral, el fracaso de la formación de la personalidad, y ofrece una clave crítica para las manifestaciones de la modernidad. Considérense a continuación con cierto detenimiento estos dos rasgos: el carácter activo y el elemento crítico de la cultura.

\subsection{Cultura como actividad}

Una determinación del concepto aparece en el breve ensayo de 1908 "De la esencia de la cultura". La cultura remite allí primariamente a un tipo de praxis, es decir una actividad consciente. Al definirla como algo llevado a cabo por la conciencia, Simmel (2008) le adscribe dos factores esenciales y recíprocamente determinantes: los medios y los fines. Sin embargo, no toda posición teleológica es una actividad cultural. Para satisfacer los requisitos de la cultura, la actividad consciente debe poseer un cierto tipo de finalidad y apuntar correspondientemente a un cierto tipo de resultado. En la cultura, el emplazamiento teleológico aspira a la realización de "algo" ya profundamente contenido dentro de las "relaciones estructurales y fuerzas motrices" del objeto. Este "algo" existe como una predisposición latente, como un impulso cuya concreción se encuentra allende el conocimiento conceptual no solo porque aún no ha devenido existente, pero también porque sus dinámicas no se encuentran gobernadas por la causalidad y por tanto no pueden desplegarse "naturalmente". Simmel encuentra tal impulso solo en la humanidad, y por ello "solo el hombre es auténtico objeto de la cultura" (SIMMEL 2008: 206). Desde la perspectiva de su finalidad, la cultura es pues una actividad humana que se dirige a la realización del alma con arreglo a un plan idealmente desplegado:

O, expresado de otra manera, cultura es el camino desde la unidad cerrada, a través de la multiplicidad cerrada, hasta la unidad desarrollada. Pero, sea como fuere, solo puede tratarse del desarrollo hacia un fenómeno que está instalado en las fuerzas nucleares de la personalidad, un fenómeno, por así decirlo, que está esbozado en ella misma como su plan ideal. (SIMMEL 2008: 99)

Tal como lo había afirmado en Filosofía del dinero (SIMMEL 2013), una acción es cultural tan pronto como produce o refuerza una personalidad. De hecho, la actividad 
García ChICOTE, F. M. - El concepto de cultura en la ensayística de Georg Simmel

cultural se concibe con arreglo al efecto que tiene sobre la "totalidad interior" del individuo, que constituye su omega:

La medida que determina el rango de cada una de nuestras realizaciones o receptividades, bajo las categorías de su serie objetiva especial, no debe ser confundida con aquella otra que permite juzgar precisamente los mismos contenidos bajo la categoría de la cultura, esto es, como desarrollo de nuestra totalidad interior (SIMMEL 2008: 210)

En lo que respecta a la necesaria interdependencia de los medios y los fines como factores de la actividad, Simmel afirma que toda expresión cultural posee dos aspectos distintivos: un aspecto genérico-objetivo que está dado por las directrices objetivas del medio en el que se produce la actividad, y otro subjetivo-individual, en el que el alma, que alcanza su existencia por medio de valores, se encamina hacia la plasmación de la personalidad. La relación entre, por un lado, aspectos mediadores y objetivos y, por el otro, la valoración anímica es, de acuerdo con Simmel, fortuita, “contingente”. Así lo afirma en las líneas inaugurales de su Filosofía del dinero ${ }^{14}$ y así lo repite casi tres lustros más tarde, en su ensayo sobre Goethe:

La contingencia de la relación que existe entre nuestros actos, como meros hechos anímicos y como valores dentro de las series reales, tiene, por consiguiente, su continuación entre los primeros y la significación de su contenido para la formación de nosotros mismos como personalidad objetiva, como hechura vital cerrada. (SIMMEL 2005b: 25)

Dado que se asume así que la autoconciencia es el despliegue de impulsos subjetivos inherentes, los medios resultan elementos a la vez disruptivos y necesarios. En efecto: si bien posibilitan la existencia objetiva del alma, causan también su debilitamiento.

Varias son las implicancias que presenta una tal ponderación negativa de los medios en el proceso de humanización. Destaca ante todo la comprensión elitista, en Simmel, de que solo cierto tipo de praxis es en última instancia significativa. En su Goethe, Simmel (2005b) sostiene que hay tres tipos diferentes de hombres activos: aquellos subyugados por los medios, aquellos enceguecidos por los fines y aquellos que logran superar la "división mecánica" de los dos primeros en virtud de su entrega al despliegue de su alma. Respectivamente, el segundo y el tercer tipo los constituyen el filisteo, cuya fijación en la realidad objetiva lo conduce a actitudes "mammonistas", y el

\footnotetext{
14 "Que los objetos, los pensamientos y los acontecimientos sean valiosos no se podrá deducir nunca de su existencia y sus contenidos naturales; su orden, si establecido de acuerdo con los valores, se distancia enormemente del natural. [...] La relación entre ambos órdenes es [...] contingencia absoluta (SIMMEL 2013: 41-42).
}

Pandaemonium, São Paulo, v. 23, n. 41, set.-dez. 2020, p. 153-175 
GarCíA CHICOTE, F. M. - El concepto de cultura en la ensayística de Georg Simmel

artista. El primer tipo es el trabajador, cuya única preocupación es cómo llegar a fin de mes (SIMMEL 2005b: 19). La superación por parte del arte de estos dos tipos subjetivos, que de acuerdo con Simmel parecen opuestos, pero que en realidad mantienen entre sí una identidad última, es empero no dialéctica. A diferencia del impulso del juego en Schiller, la actividad artística no se halla demandada y gobernada por la razón, ni exige necesidad y universalidad. La "genialidad" del artista consiste en su capacidad innata para superar las disposiciones alienadas encarnadas en los otros tipos, a pesar e independientemente de ellos.

En íntima conexión con este tratamiento subjetivista del genio artista emerge en Simmel una serie de tentativas para definir un tipo de actividad individual que prescinda del polo objetivo corruptor. Esto se manifiesta inequívocamente a partir de 1909, en la breve reseña que le dedica a El séptimo anillo, el poemario de Stefan George publicado dos años antes. Allí, Simmel afirma haber encontrado el indicio de una nueva subjetividad general cuyos despliegue solipsista, "fuerza" y "esencia absoluta" anuncian la posibilidad de regir la vida con estricto arreglo a la valorización subjetiva del mundo, sin que dicha valorización conduzca necesariamente a la enajenación objetiva de valores (SIMMEL 2001: 54). Adquiere importancia decisiva para toda esta línea teórica que gana paulatinamente lugar en la ensayística del filósofo -y se consolida en los últimos años en trabajos como Rembrandt o "El conflicto de la cultura moderna"- la adopción de una versión fichteana del concepto de Stimmung (estado de ánimo), entendido como autoactividad no mediada. ${ }^{15}$ De este modo, la tensión que subyace al concepto de actividad cultural y que proviene de su necesaria vinculación de dos órdenes supuestamente irreconciliables - el de los valores y el de la objetividad-se resuelve en Simmel en la afirmación de un sujeto actuante no objetivo, que, de acuerdo con Ernst Cassirer (1965: 160) en 1942, asume rasgos "místicos":

Simmel parece hablarnos el lenguaje del escéptico; pero nos habla, en realidad, en el del místico. El anhelo secreto de toda mística no es otro, en efecto, que el de sumirse pura y exclusivamente en la esencia del yo, para descubrir en ella la esencia de Dios. Cuanto se interfiere entre el yo y Dios es considerado para ella como un tabique de separación.

\footnotetext{
${ }^{15}$ Nótese que la crítica despiadada del concepto de cultura que el joven Lukács lleva a cabo en 1911, crítica a la que nos hemos referido en la sección anterior, arraiga en un examen de esta variante fichteana de la Stimmung: para Lukács, la "libertad total" que se pretende alcanzar al afirmar que "“todo es estado de ánimo': maravillosa, sublime libertad del alma, su dominio sobre todas las cosas, absorción de lo existente dentro del alma, que es lo único que vive" es en verdad la "más rigurosa esclavitud" (cf. LuKÁcs 2015: 188-189).
} 
García ChICOTE, F. M. - El concepto de cultura en la ensayística de Georg Simmel

Es sugerente que para Cassirer (1965: 161-165), dicho tratamiento místico, que concibe "toda pluralidad de cosas" como un "engaño", resulte de la afirmación de la sustancialidad individual del yo en tanto punto de partida metodológico.

\subsection{Cultura como crítica}

El potencial crítico normalmente imputado a la teoría cultural simmeliana -esto es, su supuesta capacidad para iluminar y denunciar procesos modernos de alienación- puede ser visto como un correlato lógico del concepto de actividad recién expuesto, que proviene a su vez de una determinación en última instancia subjetivista de la valorización.

Para Simmel, la alienación es un empobrecimiento espiritual causado por la incapacidad del alma para recuperar valores para sí. En Filosofía del dinero, se habla de una "espiritualización de los objetos": un proceso según el cual estos "parecen realizarse según las propias fuerzas y normas” (SIMMEL 2013: 534). Así resulta una situación vital imperante en la que las expresiones subjetivas, objetivadas, "en su causalidad estricta se aproxima[n] al cosmos de las leyes naturales" (SIMMEL 2013: 511). La rigurosa vigencia de las legalidades naturales en el mundo anímico, la pérdida de conexión de los valores objetivados con los sentimientos subjetivos, la emergencia de un mundo humano racionalmente calculable, pero anímicamente insignificante... todos estos son los rasgos de un diagnóstico de la modernidad que recuerda en buena medida el análisis que el joven Lukács hace de la sociedad burguesa en La teoría de la novela, de 1916. Allí, conforme la primera formulación del concepto de "segunda naturaleza", se denuncia "un mundo presente en todas partes con inabarcable multiplicidad; un mundo cuya rigurosa ley, tanto en el devenir cuanto en el ser, se hace evidente al sujeto conocedor con necesidad, pero que, pese a sus leyes, no se ofrece como sentido al sujeto que busca fines" (LUKÁCS 1985: 329). ${ }^{16}$ Como hemos visto, la condición de posibilidad de tal enajenación anímica proviene de la existencia de los valores, es decir, del mero hecho de que estos asumen una forma objetiva que es, en virtud de su objetividad, ajena al alma. Perfílase así una diferencia sustantiva con los desarrollos del joven Marx (2010) en torno al problema de

\footnotetext{
${ }^{16}$ En una retrospectiva acerca de su acaso más famoso ensayo de juventud, el propio Lukács (1985: 282) reconoce la impronta de "[Wilhelm] Dilthey, Simmel y Max Weber" en su temprana "recusación [...] de la sociedad burguesa", "utópica" en la medida en que "ni siquiera en el plano del pensamiento concebía yo entonces mediaciones entre la toma de posición subjetiva y la realidad objetiva" (1985: 282).
} 
GarCíA CHICOTE, F. M. - El concepto de cultura en la ensayística de Georg Simmel

la alienación: para este, la prioridad ontológica del objeto descarta la posibilidad de una subjetividad no objetiva, al punto de que se lee en los así llamados Manuscritos económico-filosóficos que "un ser no objetivo" es un "Unwesen", lo que significa tanto un "no ser" como un "monstruo". ${ }^{17}$ En Simmel, en cambio, la monstruosidad, el desarrollo distorsivo de las capacidades individuales tiene su origen en la interferencia objetiva de un ser que se concibe, al menos postulativamente, radicalmente subjetivo.

Los valores encapsulados en los objetos -imposibilitados de fluir de vuelta al alma que una vez los expresó, y por lo tanto imposibilitados de servir a la autoconcienciaganan autonomía; aseguran su existencia al apoyarse en las legalidades objetivas de sus vehículos; crecen en volumen a causa de la expresividad anímica ilimitada; gradualmente, borran de sus aspectos visibles toda marca que podría reconducirlos al alma. Independizados de su alfa y omega, los valores terminan animando sus propias existencias, empoderando así a los objetos con una energía que en esencia estos no poseen. Por el otro lado, el alma fracasa en reconocer los valores en objetos, bien porque el certificado de origen ha sido borrado, bien porque las facultades recuperadoras relativamente lentas del alma no pueden ir a la par de la rápida profusión de objetos en la modernidad. En "Crisis de la cultura", un ensayo de 1915 que indaga sobre los efectos paliativos que la guerra en Europa puede tener sobre la alienación cultural, este problema se resume del siguiente modo:

Los objetos -que al mismo tiempo cargan y son cargados por la vida cultural- siguen, precisamente cuanto más refinados y a su modo perfeccionados estén, una lógica inmanente que de ninguna manera se inserta siempre dentro de aquel desarrollo de los sujetos que vuelve sobre sí mismo del modo en que debería, dado el sentido de todas las formaciones culturales en tanto tales. Innumerables objetivaciones del espíritu, obras artísticas y formas sociales, instituciones y conocimientos, se nos enfrentan como imperios administrados según leyes propias que pretenden tornarse contenido y norma de nuestra existencia individual; esta, sin embargo, no sabe emprender nada correcto con tales objetivaciones, e incluso muy a menudo las percibe como cargas y fuerzas antagonistas. Pero no solo esta extrañeza cualitativa se halla entre lo objetivo y lo subjetivo de las culturas superiores; sino también esencialmente el carácter cuantitativamente ilimitado con el que se suceden libro tras libro, descubrimiento tras descubrimiento, obra de arte tras obra de arte... una por así decirlo infinitud informe que se acerca a los individuos con la pretensión de ser absorbida. (SIMMEL 2000: 191)

Por tanto, el alma es (1) empobrecida por su pérdida constante de valores y (2) cautivada por el crecimiento constante de objetos animados. Cuanto más asumen los

\footnotetext{
${ }^{17}$ Cf. Marx (2010: 199). Debo la indicación de la complejidad semántica del término "Unwesen" y la riqueza que éste adquiere en la obra de Marx a Miguel Vedda.
}

Pandaemonium, São Paulo, v. 23, n. 41, set.-dez. 2020, p. 153-175 
García ChICOTE, F. M. - El concepto de cultura en la ensayística de Georg Simmel

objetos una existencia autónoma, tanto más deviene el alma dependiente de ellos y, consecuentemente, tanto menos consciente de sí misma. El alma adquiere de esta manera un modo de existencia propio de una cosa, y la cosa un modo de existencia propio del alma: cosificación y fetichismo. En "El concepto y la tragedia de la cultura", de 1911, Simmel entiende el fetichismo como un problema general de la expresividad del alma:

El "carácter de fetiche" que Marx adscribe a los objetos económicos en la época de producción de mercancías es solo un caso peculiarmente modificado de este destino general de nuestros contenidos culturales. Estos contenidos están bajo la paradoja $-\mathrm{y}$, con una "cultura" creciente, cada vez más- de que, ciertamente, han sido creados por sujetos y están determinados para sujetos, pero en la forma intermedia de la objetividad que adoptan más allá y más acá de estas instancias siguen una lógica evolutiva inmanente y, en esta medida, se alejan tanto de su origen como de su fin. No son necesidades físicas las que entran en cuestión a este respecto, sino realmente solo necesidades culturales. (SIMMEL 2008: 116)

Si la condición de posibilidad para el mundo alienado es la brecha entre sujeto y objeto, su causa histórica ha de buscarse en la emergencia y el desarrollo de la división del trabajo. Simmel (2013: 540) afirma que la formación de una personalidad, de una trabazón cerrada y armoniosa de las capacidades individuales depende en buena medida de la relación multilateral, anímica y cerrada que el individuo posea con el objeto en cuyo proceso productivo plasma su interioridad: "la totalidad interna del Yo se establece en reciprocidad con la cerrazón y la redondez de las tareas vitales”. Ahora bien, la especialización genera formas de existencia "inadecuadas" entre el productor -que experimenta un aumento unilateral de las "energías y habilidades psico-físicas"- y el producto -cuya redondez no expresa ninguna cerrazón anímica-. Así, el producto que resulta de la cooperación de desarrollos unilaterales "no puede buscar su significación ni en la proyección de una subjetividad ni en el reflejo que emite en el alma creadora como expresión de esta, sino que únicamente puede encontrarla como resultado objetivo en su apartamiento del sujeto" (SIMMEL 2013: 540).

"División del trabajo" es pues aquello que "separa a la persona creadora de la obra creada" (SIMMEL 2013: 543-544). El "sentido amplio" de esta definición revela los lineamientos fundamentales del concepto y sus implicancias para una teoría de la alienación: aunque no la llame así, Simmel se refiere en rigor a la forma histórico-concreta de la división técnica del trabajo cada vez que habla del concepto general de división del trabajo. La división técnica del trabajo, esto es la cada vez más planificada parcialización de trabajos conforme mecanismos de espacialización y temporalización que apuntan a un incremento cada vez mayor de la productividad y que, de una parte, echan históricamente 
García ChICOTE, F. M. - El concepto de cultura en la ensayística de Georg Simmel

por tierra la relación individual, propia de la artesanía, entre el productor y el producto y, por otro, tienden a una cada vez mayor concentración de los medios de producción por parte de pocos individuos, es concebida en Filosofía del dinero como distorsión histórica inevitable de la armonía individual. ${ }^{18}$ Por ello puede, por un lado, afirmar, al menos postulativamente, la creación humana sin división del trabajo ${ }^{19} \mathrm{y}$, por el otro, equiparar sin más la división técnica del trabajo con la alienación capitalista.

En suma, la aproximación de Simmel al problema de la división del trabajo parece no superar, a pesar del valor de sus minuciosos análisis individuales, el nivel fundamental de aquella contradicción denunciada por Marx en la que la "conciencia burguesa" cae cada vez que aborda la cuestión de la división planificada de las tareas: por un lado, ella "celebra la división manufacturera del trabajo, la anexión vitalicia del obrero a una operación parcial y la subordinación incondicional de los obreros parciales al capital"; por el otro, "denuncia con igual vigor todo control y regulación sociales [...], en los que ve un cercenamiento de los sacrosantos derechos de propiedad, de la libertad y de la 'genialidad' -que se determina a si misma- del capitalista individual'” (MARX 1985: 434). Ciertamente, todo el núcleo argumentativo de Simmel se funda no en la celebración, sino en la crítica de la parcialización de las tareas productivas; pero su punto de partida es el mismo individualismo metodológico que posibilita la hipocresía señalada por Marx, la abstracta suposición de un individuo privado "que se determina a sí mismo" y cuya felicidad depende de su - postulativa- sustracción de la división técnica del trabajo, que Simmel concibe como división social del trabajo tout court. A los efectos de nuestra exposición, la signatura saliente de este concepto de división del trabajo es el pesimismo tan característico de la sociología alemana y la vía libre a la búsqueda de soluciones metafísicas (la Primera Guerra Mundial es la que provee tal posibilidad para Simmel): el individuo privado, que cree encontrar su más fiel figura en modos propios de la artesanía, se propone como única y a la vez históricamente superada configuración subjetiva capaz de alcanzar el desarrollo pleno y armonioso de sus capacidades; no se divisa ningún

\footnotetext{
18 Acerca del modo en que la economía política clásica confunde la relación entre la forma histórica de la división técnica del trabajo con la división social del trabajo véase la historia del pensamiento económico de Isaac Illich Rubin de 1929. El concepto de división técnica del trabajo fue parcialmente tomado de allí (RUBIN 1979: 177-185).

${ }^{19}$ Remítase, para una contraposición, a la obra de madurez de Lukács, en la que división social del trabajo es una determinación ontológica del ser humano y aparece como tal ya en el esquema básico de trabajo (cf. LUKÁCS 2004: 43).
} 
GarCíA CHICOTE, F. M. - El concepto de cultura en la ensayística de Georg Simmel

potencial emancipador de las nuevas formas de subjetividad históricamente nacidas de la gran empresa capitalista. ${ }^{20}$

La especialización da rienda suelta entonces a un conflicto entre sujeto y objeto que asume en la ensayística del filósofo los términos de "preeminencia" de un "espíritu" o una "cultura" objetiva sobre un "espíritu" o una "cultura" subjetiva: tal sería la marca distintiva de la modernidad. Dicha preeminencia tiene dos características. De una parte, ocurre una aceleración en la "economía dineraria", vinculada con la modernidad en general y con sus formas idiosincrásicas de vida urbana. Por el otro lado, la reificación del alma lleva al desarrollo hipertrofiado de la racionalidad, que Simmel equipara con la noción neokantiana de entendimiento. El alma, esencialmente irracional, se opone a este ámbito de lo racional, vinculado no con valores, sino con la legalidad objetiva de las formaciones. Esta tensión entre aspectos contrarios constitutivos del individuo moderno se tematiza en "Las grandes ciudades y la vida del espíritu", de 1903, en términos de capas profundas y capas superficiales. Al hipertrofiarse, el entendimiento cubre con una envoltura impenetrable la verdadera naturaleza del individuo, su interioridad, y malogra cualquier comunicación de esta con el mundo externo. La incapacidad del alma para estrechar lazos con el afuera provoca una excesiva individuación y una exacerbación de las pasiones. En la ciudad moderna, dice allí Simmel (1986: 9),

se ofrece a nosotros, bajo la forma de edificios, de establecimientos de enseñanza, en los milagros y el confort de las técnicas de transporte, en las formas de la vida social y en las instituciones visibles del Estado, una abundancia a tal grado aplastante de espíritu cristalizado, despersonalizado, que el individuo no consigue, por así decirlo, mantenerse de cara a él. Por un lado, la vida se le vuelve al individuo infinitamente fácil, pues de todas partes se le ofrecen incitaciones, estímulos, ocasiones de colmar el tiempo y la conciencia, que lo arrastran en su corriente al grado de dispensarlo de tener que nadar por sí mismo. Pero por otro lado la vida se compone de cada vez más elementos de estos, de esos espectáculos impersonales que sofocan los rasgos verdaderamente individuales y distintivos; de ahí que los elementos personales deban, para subsistir, hacer un esfuerzo extremo; es preciso que lo exageren, aunque solo sea para seguir siendo audibles, en primer lugar para sí mismos.

De este modo, el fetichismo y la reificación se conciben como derivados normales de una complejización histórica igualmente normal de la actividad y de su inevitable status

\footnotetext{
${ }^{20}$ Ilustrativo para este punto, que constituye un verdadero parteaguas entre Simmel y varios de sus "herederos" (como por ejemplo György Lukács y Siegfried Kracauer), es el siguiente aforismo de 1915: "Es la vergüenza de las 'personas cultas' modernas -o lo era al menos hasta la vivencia de esta guerra- el hecho de que si bien están formadas en la ciencia natural como en la histórica, esto es que su interés responde con éxito delicado, amplio y profundo a lo pasado, a lo efectivo, a lo intemporal-, han dejado a la socialdemocracia y a los proletarios la pasión por el futuro, el conocimiento, el bosquejo de todo lo que se puede llamar "determinación del hombre"' (SIMMEL 2004: 132).
} 
GARCíA CHICOTE, F. M. - El concepto de cultura en la ensayística de Georg Simmel

contradictorio (esto es, el hecho de que los valores se apoyen, para su existencia, en elementos objetivos cuya legalidad les es ajena). Este es también el caso al interior de la esfera de la cultura, el dominio en el que la actividad ha de asistir a la autoconciencia del alma. Ciertamente, el conflicto entre valor y objeto puede ser allí atenuado en la medida en que la porción anímica en ambos es, por así decirlo, mayor. Sin embargo, en este ámbito, la productividad anímica asume formas que en virtud de su preexistencia son siempre capaces de actuar como vehículos distorsionadores y contribuyen así a la fosilización del alma y, como Simmel lo dice en 1911, a la emancipación de "contenidos culturales", que

\begin{abstract}
siguen por último una lógica independiente de su fin cultural y que los conduce cada vez más lejos de esta, sin que el camino del sujeto sea eximido de todos estos contenidos que se han tornado inadecuados cualitativa y cuantitativamente. Antes bien, puesto que este camino, en tanto que cultural, se encuentra condicionado por el tornarse autónomos y objetivos de los contenidos anímicos, surge la trágica situación de que la cultura ya esconde realmente en sí, en su primer momento existencial, aquella forma de sus contenidos que está determinada a hacer sin guía y de manera discrepante, a desviar, a gravar, a su ser interno [...] como en virtud de una inevitabilidad inmanente. (SIMMEL 2008: 121)
\end{abstract}

Visto desde su fundamento, el proceso cultural de la modernidad, obturado por la división del trabajo y apoyado en su propia lógica, encierra un destino trágico: la cultura ya no forma la personalidad, sino que avanza irremediablemente hacia la ruina.

\title{
4 Conclusión
}

Las páginas precedentes han intentado dar con el concepto de cultura en la ensayística de Simmel. Para ello, se ha procurado definir breve y esquemáticamente la noción al interior del campo intelectual en cuyo seno arraigaron las formulaciones teóricas del filósofo. Se señaló el rol decisivo desempeñado por tal noción en el proceso de autoconciencia de la idiosincrásica burguesía alemana. Al vincularse con otros conceptos axiológicos tales como formación y personalidad, la cultura describía el ámbito de perfectibilidad del ser humano; un ámbito por cierto "puramente espiritual" y contrapuesto tanto a la naturaleza como a las esferas sociales no destinadas a la formación del individuo. Estas últimas recibían el nombre de civilización.

En Simmel, el concepto mantiene estos trazos generales a la vez que se complejiza. Por un lado, se lo subordina a la lógica general de la actividad humana, con lo que se logra desdibujar aquella diferencia entre cultura espiritual y civilización 
García ChICOTE, F. M. - El concepto de cultura en la ensayística de Georg Simmel

material. Por el otro, pierde su inequívoco carácter positivo y recupera el pesimismo rousseauniano: se vuelve así fecundo para explicar procesos propios de la alienación moderna. Sin embargo, la teoría cultural de Simmel revela en su fundamento una teoría del valor que se acerca al giro subjetivista de los primeros marginalistas y la escuela austríaca: destaca una noción de sujeto en tanto prius ontológico que se revela como individualismo metodológico. Se despliega así un esquema básico de actividad en el que entran en colisión irremediable elementos subjetivos y objetivos. La ponderación negativa de los medios en el proceso de valorización conduce a la búsqueda de modos existenciales puramente subjetivos, que se aproximan a la mística.

El individualismo metodológico propio de la segunda mitad del siglo XIX -y asociado en el pensamiento filosófico a la disolución del hegelianismo- se manifiesta asimismo en la piedra angular de la explicación simmeliana de la alienación moderna: su concepto de división del trabajo. Se ha indicado que Simmel concibe la parcialización planificada de la producción en términos negativos, pues solo ve en ella la forma histórico-concreta de la división técnica del trabajo. La especialización acaba con el propósito de la cultura, la formación de personalidades individuales.

Así, la teoría cultural de Georg Simmel reviste, a pesar de sus idiosincrásicos impulsos críticos, insoslayables trazos apologéticos, puesto que deriva metodológicamente su fuerza analítica de una concepción ahistórica y subjetivista de la relación sujeto-objeto. Tal concepción coloca como alfa y omega de sus intereses y presupuestos un individuo particular burgués autodeterminado: la caída de este se considera entonces como la ruina de la cultura humana.

\section{Referencias bibliográficas}

AdORno, T. W. Dialéctica negativa. Madrid: Editora Nacional, 2002.

Adorno, T. W. Minima Moralia: reflexiones desde la vida dañada. Madrid: Taurus, 2001.

BAECKER, D. K. In: BARCK, K. (ed.). Ästhetische Grundbegriffe: Historisches Wörterbuch in sieben Bänden. Stuttgart: J. B. Meltzer, 2002, 510-556. v. 3.

BECHER, H. J. Georg Simmel en Estrasburgo (1914-1918). Tres entrevistas con un testigo: Charles Hauter (1888-1981). Revista Colombiana de Sociología, n. 31, 2008, p. 69-81.

BOLLENBECK, G. Bildung und Kultur: Glanz und Elend eines deutschen Deutungsmusters. Frankfurt: Suhrkamp, 1996.

Brecht, B. Prosa 3. Sammlungen und Dialogen. Berltolt Brecht-Werke, Band 18. Berlin: Suhrkamp, 1995.

Brinkmann, H. Methode und Geschichte: Die Analyse der Entfremdung in Georg Simmels „Philosophie des Geldes“. Gießen: Focus, 1974. 
García Chicote, F. M. - El concepto de cultura en la ensayística de Georg Simmel

CAntó Milà, N. A Sociological Theory of Value. Georg Simmel's Sociological Relationism. Bielefeld: Transcript, 2005.

CASSIRER, E. Las ciencias de la cultura. México, DF: Fondo de Cultura Económica, 1965.

Christian, P. Einheit und Zwiespalt. Zum hegelianisierenden Denken in der Philosophie und Soziologie Georg Simmels. Berlin: Duncker \& Humblot, 1978.

Dilthey, W. Vida y poesía. México, DF: Fondo de Cultura Económica, 1950.

ELIAS, N. El proceso de la civilización. México, DF: Fondo de Cultura Económica, 2009.

FRISBY, D. Fragmentos de la modernidad: Teorías la modernidad en la obra de Simmel, Kracauer y Benjamin. Madrid: Visor, 1992.

FRISBY, D. Introducción. In: SiMMEL, Georg. Filosofía del dinero. Madrid: Capitán Swing, 2013, p. 9-29.

GangAS, S. Simmel, Marx and the Radical Concept of Life: A Hegelian Approach. Dissonância, v. 3, 2019, p. 1-28.

GAY, P. Weimar Culture: The Outsider as Insider. New York: W. W. Norton \& Company, 1992.

HABERMAS, J. Georg Simmel on Philosophy and Culture: Postcript to a Collection of Essays. Critical Inquiry, v. 22, n. 3, 1996, p. 403-414.

HEINRICH, M. Die Wissenschaft vom Wert. Münster: Westfällisches Dampfboot, 2017.

JAUSS, H. R. Las transformaciones de lo moderno: estudios sobre las etapas de la modernidad estética. Madrid: Visor, 1995.

KovaL, M. I. Vocación y Renuncia: La novela de formación alemana entre la Ilustración y la Primera Guerra Mundial. Buenos Aires: Editorial de la Facultad de Filosofía y Letras de la Universidad de Buenos Aires, 2018.

Kroeber, A. L.; Kluckhohn, C. Culture. A Critical Review of Concepts and Definitions. Cambridge: The Peabody Museum of American Archaeology and Ethnology, 1952. (Série Papers of the Peabody Museum of American Archaeology and Ethnology v. 47, n. 1).

LUKÁCS, G. Los fundamentos ontológicos del pensamiento y la acción humanos. In: LUKÁCS, G. Ontología del ser social: el trabajo. Buenos Aires: Herramienta, 2004, p. 35-55.

LUKÁCS, G. Acerca de la pobreza del espíritu y otros escritos de juventud. Buenos Aires: Gorla, 2015.

LUKÁCS, G. El alma y las formas: la teoría de la novela. Barcelona: Grijalbo, 1985.

LUKÁCS, G. El asalto a la razón. Barcelona: Grijalbo, 1972.

MARCUSE, H. Cultura y sociedad. Buenos Aires: Sur, 1970.

MARX, K. El capital. México, DF: Siglo XXI, 1985. v. 1.

MARX, K. Manuscritos económico-filosóficos de 1844. Buenos Aires: Colihue, 2010.

MeAD, G. H. Philosophie des Geldes. In: RAMmstedt, O. (ed.). Georg Simmels Philosophie des Geldes. Aufsätze und Materialien. Frankfurt: Suhrkamp, 2003, p. 300-304.

Mendelssohn, M. Was ist Aufklärung?. In: BAHR, E. (ed.). Was ist Aufklärung? Thesen und Definitionen. Stuttgart, Reclam, 1974, p. 3-8.

Merton, R. K. Civilization and Culture. Sociology and Social Research, v. 21, 1936, p. 103-113.

NOLTE, P. Georg Simmels Historische Anthropologie der Moderne. Rekonstruktion eines Forschungsprogramms. Geschichte und Gesellschaft, v. 24, n. 2, 1998, p. 225-247.

PAul, A. T. Wert und Wertphilosophie. In: MÜLleR, H. P.; REITZ, T. (eds.). Simmel-Handbuch. Frankfurt: Suhrkamp, 2018, p. 596-603.

PETER, L. Élan vital, Mehr-Leben, Mehr-als-Leben. Lebensphilosophische Aspekte bei Henri Bergson und Georg Simmel. In: PETER, L. Umstrittene Moderne: Soziologische Diskurse und Gesellschaftskritik. Wiesbaden: Springer, 2016, p. 67-127. 
GarcíA ChICOTE, F. M. - El concepto de cultura en la ensayística de Geors Simmel

REHBERG, K. S. Kultur, subjektive und objektive. In: MÜLLER, H. P.; REITZ, T. (eds.). SimmelHandbuch. Frankfurt: Suhrkamp, 2018, p. 328-334.

RICKERT, H. Ciencia cultural y ciencia natural. Buenos Aires: Espasa Calpe, 1943.

Rubin, I. I. The Austrian School. In: DAY, R. B.; GAIDO, D. F. (eds.). Responses to Marx's Capital. Leiden: Brill, 2017, p. 430-447.

RUBIN, I. I. A History of Economic Thought. Londres: Ink Links, 1979.

Schmoller, G. Simmels Philosophie des Geldes. In: Rammstedt, O. (ed.). Georg Simmels Philosophie des Geldes. Aufsätze und Materialien. Frankfurt a/M, Suhrkamp, 2003, 282-289.

SiMMEL, G. Aufsätze und Abhandlungen 1909-1918. Band I. Gesamtausgabe v. 12. Frankfurt: Suhrkamp, 2001.

SIMMEL, G. Aufsätze und Abhandlungen 1909-1918. Band II. Gesamtausgabe v. 13. Frankfurt: Suhrkamp, 2000.

SIMMEL, G. Briefe 1880-1911. Gesamtausgabe v. 22. Frankfurt: Suhrkamp, 2005a.

Simmel, G. De la esencia de la cultura. Buenos Aires: Prometeo, 2008.

SIMMEL, G. Filosofía del dinero. Madrid: Capitán Swing, 2013.

SIMMEL, G. Goethe. Buenos Aires, Prometeo, 2005b.

SiMmel, G. Las grandes ciudades y la vida del espíritu. Cuadernos Políticos, número 45, eneromarzo de 1986, p. 5-10.

SIMMEL, G. Miszellen, Glossen, Stellungnahmen, Umfrageantworten, Leserbriefe, Diskussionsbeiträge 1889-1918. Anonyme und pseudonym Veröffentlichungen 18881920. Gesamtausgabe v. 17. Frankfurt: Suhrkamp, 2004. 